\title{
A importância da espiritualidade no tratamento de pacientes oncológicos: uma revisão de literatura
}

\section{The importance of spirituality in the treatment of oncology patients: a literature review}

\author{
Paulo de Tarso Xavier Sousa Junior ${ }^{1}$, Selena Mesquita de Oliveira Teixeira² \\ 1 - Faculdade Integral Diferencial - Facid |Wyden, Teresina, PI, Brasil. \\ 2 -Universidade de Fortaleza - UNIFOR, Fortaleza, CE, Brasil.
}

paulo_juniorpio@hotmail.com

\section{RESUMO}

Introdução: diante da descoberta de um câncer, comumente, as pessoas tendem a fazer novas representações a respeito de vários temas que envolvem a sua existência, considerando, sobretudo, a possibilidade de estarem mais próximo a morte. Um dos meios que o ser humano encontra para iniciar e prosseguir o tratamento contra o câncer é através da força que encontra em sua espiritualidade. Objetivo: analisar as discussões científicas existentes sobre como a espiritualidade auxilia na adesão das pessoas com câncer ao tratamento proposto e sobre o fortalecimento emocional diante do quadro de adoecimento. Método: a seleção dos artigos foi realizada nas seguintes bases de dados: Google Scholar/Acadêmico, SciELO, PePsic, BVS Brasil. Foram usados os seguintes descritores: câncer; religião e espiritualidade; espiritualidade e câncer. A bibliográfica utilizada compreende os anos de 2001 a 2017, perfazendo um total de vinte e cinco publicações dentre artigos, dissertações e teses. Resultados: como resultados as referências trazem a espiritualidade um fator que proporciona uma ressignificação dessa experiência para o sujeito, fazendo-o encarar a doença com mais tranquilidade e esperança por meio da força que este fator propicia diante do quadro de um adoecimento repleto de tabus. Além disso, a compreensão do fator espiritualidade permite um melhor acolhimento por parte do profissio-

Palavras-chave: Câncer; Espiritualidade; Religião; Fatores de proteção. nal para com o paciente, já que esse passa a ter uma postura mais empática, compreendendo os suas questões. Considerações finais: a espiritualidade se torna um ponto de suma importância dentro do cenário oncológico uma vez que a mesma favorece para o desenvolvimento da resiliência em cada sujeito, promovendo qualidade de vida dentro do tratamento.

\section{ABSTRACT}

Introduction: when cancer is discovered, people tend to create new representations about various topics that involve their existence, considering, above all, the possibility of being closer to death. One of the ways through which human beings initiate and continue treatment against cancer is to find strength in spirituality. Objective: the aim of the present study was to analyze existing scientific discussions about how spirituality helps treatment adherence of people with cancer and emotional strengthening in the context of illness. Method: articles were selected from the following databases: Google Scholar/ scholar, SciELO, PePsic, and BVS. The following descriptors were used: Cancer; Religion and spirituality; Spirituality and cancer. The selected literature was published between 2001 and 2017, totaling twenty-five publications among articles, dissertations and theses. Results: the literature presents spirituality as a factor that helps subjects resignify this experience and cope with the disease with more tranquility and hope based on the strength that it provides them when faced with the condition of an illness filled with taboos. Moreover, understanding the spirituality factor allows for better acceptance by professionals to the patient, since this becomes a more empathetic posture, understanding their

Keywords: fears. Final considerations: spirituality becomes a point of utmost importance within the oncology scenario, because it favors the development of resilience in each subject, promoting quality of life within the treatment. 


\section{INTRODUÇÃO}

O câncer se caracteriza como uma doença onde células se multiplicam de forma desordenada e podem chegar a atingir órgãos do corpo humano. ${ }^{1}$ Os pesquisadores não apresentam um consenso acerca das causas exatas do câncer, porém muitos estudos apontam que causas genéticas e fatores externos como o uso do cigarro e hábitos de vida não saudáveis são apontadas como fatores que contribuem para o seu surgimento. $^{2}$

De acordo com a Organização Mundial da Saúde (OMS), ${ }^{3}$ em 2015, 8,8 milhões de mortes foram ocasionadas do câncer, sendo o mesmo considerado como a segunda principal causa de morte no mundo. A cada seis mortes, uma tem como causa o câncer. O Instituto Nacional do Câncer (INCA) estima entre 2016 e 2017 a ocorrência de cerca de 600 mil casos novos de câncer no Brasil, destacando-se o câncer de pele não melanoma (aproximadamente 180 mil casos novos). Também se estima a ocorrência em cerca de 420 mil casos novos de câncer. ${ }^{4}$

O câncer, seu prognóstico e tratamento podem representar para alguns indivíduos uma ameaça à sua saúde e a integridade do corpo, oferecendo uma possibilidade de morte, deste modo, impõe tanto ao paciente, quanto a família um grande desafio para o seu enfrentamento. $\mathrm{O}$ câncer atualmente é uma das doenças que causa uma grande taxa de mortalidade gerando sofrimento de diversas ordens entre os pacientes e seus familiares. ${ }^{5,6}$

Diante da diversidade da subjetividade humana o câncer sofre modificações nas crenças e nas compreensões dos sujeitos uma vez que o entendimento sobre o mesmo é influenciado de acordo com a realidade de cada ser. Além disso, ele pode chegar a causar influencias diretas e indiretas não só no paciente mas como também em amigos e familiares próximos a pessoa diagnosticada. Dessa forma o câncer traduz uma série de significações nas pessoas causando reações adversas como o receio e até mesmo em outros casos força e superação. ${ }^{7}$

O diagnóstico de câncer acaba gerando nas pessoas um sentimento de estar mais próximo da morte, uma vez que os tratamentos utilizados são vistos como dolorosos e que causam grande fragilidade ao corpo, a medida que se apropriam de crenças como de ser uma doença incurável. ${ }^{8}$ Desse modo, se faz necessário um olhar atento para a subjetividade e a particularidade de cada sujeito para a construção de um tratamento seguro e eficaz, mas acima de tudo compreendendo os fatores que contribuem para a sua aceitação.

Atualmente existem muitas discussões a respeito de fatores que auxiliam a adesão de pacientes no tratamento do câncer, sendo um deles a religião e a espiritualidade. No Brasil, aproximadamente $86,6 \%$ da população se declara como cristã, sendo $64,6 \%$ católicos e $22,2 \%$ evangélicos e apenas $8 \%$ expressam não ter alguma religião. Portanto a fé e a crença em um Deus se torna um ponto em comum entre os diversos brasileiros, porém não se deve confundir os termos religião e espiritualidade. ${ }^{9}$

Inicialmente se faz necessário a distinção do termo espiritualidade e religião, visto que muitas vezes essas terminologias são consideradas iguais, entretanto possuem significados diferentes. Alguns autores descrevem o termo religião, do latim religião, como a conexão que une um Deus ao homem ${ }^{10}$, pois afirmam que a religião é considerada como algo mais específico ligado a uma serie de crenças $^{11}$; já a espiritualidade se refere a algo em que o indivíduo se apropria para dar sentido a sua existência, atribuindo essa tarefa a alguma divindade ou poder supremo. ${ }^{12}$

É possível compreender que as religiões trazem determinados ritos e preceitos a serem seguidos. As pessoas acreditam em um Deus, que produz a força necessária para que cada um consiga superar suas adversidades. A religião fornece subsídios que fortalece a fé, seja por meio de imagens, orações e afins, as quais deixam evidentes a sua devoção. ${ }^{13}$

A espiritualidade trás uma grande importância dentro do conceito de saúde, já que ela é uma dimensão da expressividade humana que possibilita uma melhora em seu modo de vida. Ela objetiva o sujeito a procurar formas e hábitos de vida que sejam saudáveis, sendo um argumento já comprovado cientificamente. ${ }^{14}$ Diante das experiências vividas a espiritualidade entra como um fator que fortalece a pessoa em situações difíceis. ${ }^{15}$ Podemos associar a espiritualidade, portanto a diversas perspectivas, como por exemplo, a formas de enfretamento a doenças. ${ }^{16}$

Durante o tratamento oncológico a espiritualidade auxilia no desenvolvimento da resiliência, uma vez que os pacientes enfrentam algumas barreiras, como por exemplo situações estressoras. ${ }^{17,18}$ Além disso, pessoas próximas e familiares dos sujeitos que estão em intervenção terapêutica recorrem a este fator para auxiliar na busca de reflexões e sentidos por conta do impacto do diagnóstico. ${ }^{19}$ 
Com base nas considerações acima este estudo apresenta como relevância científica a compreensão dos conhecimentos produzidos pela academia, fornecendo novas possibilidades de estudos e pesquisas. Já como relevância social produz para a formação de práticas mais acolhedoras e humanistas que permitem a compreensão de todos os aspectos da subjetividade do indivíduo, trazendo sua religiosidade e/ou espiritualidade como um fator de grande importância dentro do processo saúde-doença. Dessa forma, este trabalho tem por objetivo analisar como a espiritualidade tem contribuído positivamente no tratamento de pacientes oncológicos a partir de uma revisão de literatura.

\section{MÉTODO}

O trabalho em questão apresenta uma revisão de literatura, que busca reunir publicações produzidas de acordo com uma determinada temática, adotando o caráter narrativo e compreensivo, ou seja, aquele que busca desenvolver e relatar as ideias conforme luz de aportes teóricos de um assunto específico. ${ }^{20} \mathrm{~A}$ pesquisa foi realizada através dos bancos de dados como Google Scholar/Acadêmico, SciELO, PePsic, BVS Brasil identificando estudos que relatassem como a espiritualidade contribui para o tratamento oncológico. Os descritores que nortearam essa pesquisa foram: câncer; religião e espiritualidade; espiritualidade e câncer.

Realizou-se também a consulta de dados eletrônicos a respeito do câncer e a taxa de pessoas que são atingidas por ele mundialmente, através da Organização Mundial de Saúde (OMS), e no Brasil, pelo Instituto Nacional do Câncer (INCA). A pesquisa também inseriu dados do Instituto de Geografia e Estatística (IBGE) a respeito do número de brasileiros que possuem ou não alguma religião, achando, portanto, esses dados relevantes para a compreensão do objetivo proposto.

Como critérios de inclusão foram adotadas os seguintes aspectos: abranger apenas estudos em língua portuguesa, publicados em diversos periódicos eletrônicos. Além disso, as referências utilizadas datam do período que compreende os anos de 2001 a 2017. Já como critérios de exclusão ficaram de fora estudos que se tratavam de anais de eventos as quais possuíam apenas resumo, seja na modalidade simples ou expandido. Após uma busca nas bases de dados apoiada nesses critérios houve a seleção de trinta referências, sendo selecionadas posteriormente vinte e cinco para a construção deste estudo.

\section{RESULTADOS E DISCUSSÕES}

Após as buscas realizadas, conforme os aspectos metodológicos descritos acima, o estudo apresenta o quadro 1 com informações das referências, que após analise dos critérios definidos se encaixaram para a produção deste trabalho. Para isso se apresentam informações como o título, os autores, ano e o objetivo da publicação, sendo todos encontrados em revistas cientificas de formato eletrônico.

Diante dos dados do quadro 1 é possível inferir que as mais diversas modalidades de estudos são compostas por autores de variadas especialidades como Medicina, Enfermagem, Psicologia, Direito, Educação Física, Nutrição e afins. Também se torna notório um aumento gradativo em escritos da temática ao longo dos cinco últimos anos. As referências que constam nos resultados e discussões foram utilizadas apenas nesta seção, deixando de fora de outros tópicos deste artigo, como a introdução.

Os dados obtidos nos textos foram divididos em duas categorias: 1) fatores protetores no tratamento do câncer e 2) os aspectos da espiritualidade como enfrentamento no tratamento, sendo descritas a seguir.

\section{Fatores protetores no tratamento do câncer}

Apesar de o câncer ser uma enfermidade que conduz a sentimentos de angústia, tristeza e medo, trata-se de um processo que, por ser muito doloroso deve ser enfrentado pelo paciente juntamente a família. No entanto, esse enfrentamento ocasiona uma fragilidade em ambos, tornando-os mais suscetíveis ao medo das repercussões que podem resultar da doença. ${ }^{21} \mathrm{O}$ enfrentamento de alguma patologia está ligado ao sentido de extinguir ou alterar as condições de risco, buscando, dessa forma, adaptação social, melhora na qualidade de vida e um funcionamento psicológico equilibrado. ${ }^{22}$

É neste momento que os indivíduos passam a vivenciarem questões bastante delicadas. $\mathrm{O}$ câncer assim como outras doenças que podem trazer poucas possibilidades terapêuticas ofertam uma série de medos e angustias. A possibilidade de perder a vida, bem como o dia-a-dia e a chance de construir 
Quadro 1 - Relação dos materiais encontrados para a produção deste estudo.

\begin{tabular}{|c|c|c|}
\hline Título e Ano & Autor (es) & Objetivo \\
\hline $\begin{array}{l}\text { Estratégias de Enfrentamento do Câncer Adotadas por } \\
\text { Familiares de Indivíduos em Tratamento Oncológico } \\
(2016)^{21}\end{array}$ & $\begin{array}{l}\text { Mattos, K., Blomer, T. H., Campos, A. } \\
\text { C. B. F., \& Silvério, M. R. }\end{array}$ & $\begin{array}{l}\text { O estudo busca analisar as estratégias usadas } \\
\text { pelos familiares diante o enfrentamento do } \\
\text { câncer. }\end{array}$ \\
\hline $\begin{array}{l}\text { O Conceito de Enfrentamento e Sua Relevância na } \\
\text { Prática de Psicologia }(2010)^{22}\end{array}$ & Nunes, C. M. N. S. & $\begin{array}{l}\text { Um estudo de revisão sobre o coping dentro } \\
\text { dos aspectos psicológicos da oncologia. }\end{array}$ \\
\hline $\begin{array}{l}\text { Enfrentamento e Resiliência de Pacientes em } \\
\text { Tratamento Quimioterápico e seus Familiares }(2012)^{23}\end{array}$ & Rodrigues, F. S. S; Polidori, M. M & $\begin{array}{l}\text { Analisar acerca do enfrentamento do câncer } \\
\text { de pacientes e familiares. }\end{array}$ \\
\hline $\begin{array}{l}\text { Meios de comunicação utilizados pelos pacientes: } \\
\text { informações sobre o câncer após o diagnóstico e } \\
\text { durante o tratamento }(2016)^{24}\end{array}$ & $\begin{array}{l}\text { Hey, A. P.; Caveião, C.; Motezeli, J. } \\
\text { H.; Visentin, A.; Takano, T. M., \& } \\
\text { Buratti, F. M. S. }\end{array}$ & $\begin{array}{l}\text { Investigar e descrever quais meios de } \\
\text { comunicação são usados por pacientes } \\
\text { oncológicos. }\end{array}$ \\
\hline $\begin{array}{l}\text { Estratégias de Enfrentamento utilizadas pelos pacientes } \\
\text { oncológicos submetidos a cirurgias mutiladoras } \\
(2009)^{25}\end{array}$ & Costa P.; Leite, R. C. B. O. & $\begin{array}{l}\text { Relata sobre as estratégias usadas diante de } \\
\text { cirurgias mutiladoras devido ao tratamento } \\
\text { oncológico. }\end{array}$ \\
\hline $\begin{array}{l}\text { Significado e práticas da espiritualidade no contexto } \\
\text { dos cuidados paliativos em pacientes oncológicos } \\
\text { adultos }(2011)^{26}\end{array}$ & Silva, D. I. S. & $\begin{array}{l}\text { Revisão que busca compreender sobre a } \\
\text { espiritualidade no contexto oncológico. }\end{array}$ \\
\hline $\begin{array}{l}\text { Resiliência em famílias de pessoas portadoras de } \\
\text { colostomia por câncer: um olhar a partir do sistema de } \\
\text { crenças }(2016)^{27}\end{array}$ & $\begin{array}{l}\text { Rosa, B. V. C.; Girardon-Perlini N. } \\
\text { M. O; Rosa, D. B. N.; Stamm, B.; } \\
\text { Coppetti, L. C. }\end{array}$ & $\begin{array}{l}\text { Identificação das crenças diante da resiliência } \\
\text { em casos de colostomia. }\end{array}$ \\
\hline $\begin{array}{l}\text { Espiritualidade e Resiliência em Pacientes Oncológicos } \\
(2016)^{28}\end{array}$ & $\begin{array}{l}\text { Soratto M. T.; Silva, D. M.; Zugno, P. } \\
\text { I.; Daniel, R. }\end{array}$ & $\begin{array}{l}\text { Estudo que retrata da importância da } \\
\text { espiritualidade como um fator de resiliência. }\end{array}$ \\
\hline $\begin{array}{l}\text { A importância da Integração da espiritualidade e } \\
\text { da religiosidade no manejo da dor e dos cuidados } \\
\text { paliativos }(2007)^{29}\end{array}$ & $\begin{array}{l}\text { Peres, M. F. P.; Arantes, A. C. L. Q; } \\
\text { Lessa, P. S.; Caous, C. A. }\end{array}$ & $\begin{array}{l}\text { Revisão que descreve as estratégias diante de } \\
\text { dores crônicas com relação a espiritualidade } \\
\text { e a religião. }\end{array}$ \\
\hline $\begin{array}{l}\text { Espiritualidade e câncer em pacientes submetidos a } \\
\text { tratamento quimioterápico }(2014)^{30}\end{array}$ & Cardoso, A. H. A. & $\begin{array}{l}\text { Compreender a influência da espiritualidade } \\
\text { dentro do tratamento quimioterápico. }\end{array}$ \\
\hline $\begin{array}{l}\text { O significado da religiosidade para pacientes com } \\
\text { câncer e para profissionais de saúde }(2009)^{15}\end{array}$ & Espíndula, J. A. & $\begin{array}{l}\text { Identificar com pacientes e profissionais } \\
\text { entendem a religiosidade dentro do } \\
\text { tratamento oncológico. }\end{array}$ \\
\hline $\begin{array}{l}\text { Espiritualidade na prática clínica: o que o clinico deve } \\
\text { saber? }(2010)^{31}\end{array}$ & $\begin{array}{l}\text { Lucchetti, G.; Granero, A. L.; Bassi, } \\
\text { R. M.; Latorraca, R.; Nacif, S. A. P. }\end{array}$ & $\begin{array}{l}\text { Descrição sobre o que os profissionais } \\
\text { de saúde devem compreender sobre a } \\
\text { espiritualidade no contexto clínico. }\end{array}$ \\
\hline $\begin{array}{l}\text { A importância da espiritualidade em pacientes com } \\
\text { câncer }(2015)^{32}\end{array}$ & $\begin{array}{l}\text { Pinto, A. C.; Marchesini, S.; Zugno, P. } \\
\text { I.; Zimmermann, K. G.; Dagostin, V. } \\
\text { S.; Soratto, M. T. }\end{array}$ & $\begin{array}{l}\text { Estudo que busca compreender a } \\
\text { espiritualidade como aspecto relevante no } \\
\text { tratamento do câncer. }\end{array}$ \\
\hline $\begin{array}{l}\text { A fé como fator de resiliência no tratamento do câncer: } \\
\text { uma análise do que pensam os profissionais da saúde } \\
\text { sobre o papel da espiritualidade na recuperação dos } \\
\text { pacientes }(2008)^{33}\end{array}$ & Bartolomei, M. & $\begin{array}{l}\text { Trabalho que investiga a resiliência dentro do } \\
\text { processo de doenças terminais sobre o olhar } \\
\text { dos profissionais. }\end{array}$ \\
\hline $\begin{array}{l}\text { A mulher com câncer de mama: apoio social e } \\
\text { espiritualidade }(2006)^{34}\end{array}$ & $\begin{array}{l}\text { Hoffmann, F. S.; Müller, M. C.; Rubin, } \\
\text { R. }\end{array}$ & $\begin{array}{l}\text { Compreender a importância do apoio } \\
\text { espiritual e social a mulheres com câncer de } \\
\text { mama. }\end{array}$ \\
\hline $\begin{array}{l}\text { Espiritualidade, Depressão e Qualidade de Vida no } \\
\text { Enfrentamento do Câncer: Estudo Exploratório } \\
(2015)^{35}\end{array}$ & $\begin{array}{l}\text { Miranda, S. L.; Lara e Lanna, M. A.; } \\
\text { Felippe, W. C. }\end{array}$ & $\begin{array}{l}\text { Pesquisa com o objetivo de entender as } \\
\text { relações existentes entre as dimensões } \\
\text { espirituais, depressão e qualidade de vida. }\end{array}$ \\
\hline $\begin{array}{l}\text { Fatores de risco e de proteção para câncer } \\
\text { de mama: uma revisão sistemática }(2011)^{36}\end{array}$ & $\begin{array}{l}\text { Inumaru, L. E.; Silveira, E. A.; Naves, } \\
\text { M. M. V. }\end{array}$ & $\begin{array}{l}\text { Identificar os fatores de risco e de proteção ao } \\
\text { câncer de mama. }\end{array}$ \\
\hline $\begin{array}{l}\text { Relação entre vivência de fatores estressantes e } \\
\text { surgimento de câncer de mama }(2014)^{37}\end{array}$ & Amorim, M. A. P.; Siqueira & $\begin{array}{l}\text { Busca compreender as influências do estresse } \\
\text { diante da incidência do câncer. }\end{array}$ \\
\hline $\begin{array}{l}\text { Enfrentamento religioso/espiritual em pessoas com } \\
\text { câncer em quimioterapia: revisão integrativa da } \\
\text { literatura }(2017)^{38}\end{array}$ & $\begin{array}{l}\text { Sousa, F. F. D. P. R., Melo S. M. F. F., } \\
\text { Farias, A. G. S., Oliveira, M. D. C. S., } \\
\text { Araújo, M. F. M., \& Veras, V. S. }\end{array}$ & $\begin{array}{l}\text { Revisão sobre os estudos que trazem os } \\
\text { enfrentamentos do câncer por meio da } \\
\text { espiritualidade. }\end{array}$ \\
\hline $\begin{array}{l}\text { Espiritualidade e o processo de morrer: reflexões de } \\
\text { uma equipe interdisciplinar de cuidados paliativos } \\
(2016)^{39}\end{array}$ & $\begin{array}{l}\text { Arrieira, I. C. O.; Thofern, M. B.; } \\
\text { Porto, A. R.; Amestoy, S. C.; Cardoso, } \\
\text { D. H. }\end{array}$ & $\begin{array}{l}\text { Identificar como a espiritualidade se faz } \\
\text { presente em uma equipe interdisciplinar. }\end{array}$ \\
\hline $\begin{array}{l}\text { Significados da espiritualidade para pacientes } \\
\text { com câncer em cuidados paliativos }(2017)^{40}\end{array}$ & $\begin{array}{l}\text { Benites, A. C.; Neme, C. M. B.; } \\
\text { Santos, M. A. S. }\end{array}$ & $\begin{array}{l}\text { Compreender a espiritualidade diante dos } \\
\text { cuidados paliativos ofertados no tratamento } \\
\text { do câncer. }\end{array}$ \\
\hline
\end{tabular}




\begin{tabular}{|lll|}
\hline $\begin{array}{l}\text { Espiritualidade, religiosidade e crenças pessoais de } \\
\text { adolescentes com câncer }(2015)^{41}\end{array}$ & $\begin{array}{l}\text { Souza, V. M.; Frizzo, H. C. F.; Paiva, } \\
\text { M. H. P.; Bousso, R. S.; Santos, A. S. }\end{array}$ & $\begin{array}{l}\text { Como adolescentes em tratamento de câncer } \\
\text { compreendem a espiritualidade. }\end{array}$ \\
\hline $\begin{array}{l}\text { Relação entre a esperança e a espiritualidade de idosos } \\
\text { cuidadores }(2017)^{42}\end{array}$ & $\begin{array}{l}\text { Souza, É. N.; Oliveira, N. A.; Luchesi, } \\
\text { B. M.; Gratão, A. C. M; Orlandi, F. S.; } \\
\text { Pavarini, S. C. I. }\end{array}$ & $\begin{array}{l}\text { Entender a relação entre a esperança e a } \\
\text { espiritualidade de idosos que são cuidadores. }\end{array}$ \\
\hline $\begin{array}{l}\text { O impacto da espiritualidade frente às adversidades do } \\
\text { câncer: revisão integrativa }(2016)^{43}\end{array}$ & $\begin{array}{l}\text { Rocha, R. C. N. P.; Pereira, E. R.; Sora, } \\
\text { A. B. A; Andrade Silva, R. M. C. A. }\end{array}$ & $\begin{array}{l}\text { Entender a espiritualidade dentro das } \\
\text { oncológico. }\end{array}$ \\
\hline $\begin{array}{l}\text { Câncer de mama: o apoio recebido no enfrentamentontadas no tratamento } \\
\text { da doença }(2017)^{44}\end{array}$ & $\begin{array}{l}\text { Santos, I. D. L.; Alvares, R. B.; Lima, } \\
\text { N. M.; Mattias, S. R.; Cestari, M. E. } \\
\text { W.; Pinto, K. R. T. F. }\end{array}$ \\
\hline
\end{tabular}

Fonte: Pesquisa Direta (2019)

mais projetos de vida geram sentimentos adversos, influenciando na saúde física e mental. As perdas são apresentadas como irreparáveis e a aflição diante das consequências delas assustam milhares de pessoas que se percebem dentro de um tratamento de uma doença maligna. ${ }^{33}$

O processo de enfrentamento ao câncer, visto que se trata de uma doença ameaçadora a vida, busca produzir atividades que favoreçam o bem estar dos sujeitos. Procurando reduzir o desequilíbrio causado pela doença, para que o indivíduo possa suportá-la e possa lidar positivamente consigo mesmo. ${ }^{6}$ Nessa perspectiva os sujeitos passam por uma nova fase de adaptação, visto que dependendo do tipo de câncer, o estágio e o tempo que transcorreu desde este período até o inicio do tratamento trás mudanças físicas que alteram a auto percepção. Desse modo, os sujeitos passam por uma perda da identidade que proporciona, inclusive, mudanças sociais. Esses indivíduos passam a enxergar como se não houvesse mais seu lugar na sociedade, já que o tratamento e a doença passa a ofertar restrições que antes não existiam. ${ }^{34}$

Devido a essa problemática, surgem questionamentos acerca do diagnóstico, tratamento e, principalmente, a busca por um meio de enfrentar e obter informações sobre o câncer. É essencial estudos frente a resiliência com finalidade de compreender a doença melhor e, deste modo, poder auxiliar os pacientes oncológicos destacando questões emocionais, já que estas são extremamente afetadas pelo diagnóstico de câncer. ${ }^{23}$

Além disso, outro fator de grande importância é o apoio socio-emocional que os sujeitos possuem. Conforme estudos, a força gerada por meio dos familiares, amigos e demais pessoas que fazem parte da rede social dos pacientes possibilitam a retomada de sentido da vida. Esse suporte auxilia no tratamento a ser enfrentado, possibilitando ocasiões onde os profissionais de saúde encontram uma oportunidade para dialogar sobre os próximos passos do tratamento a serem realizados. Este apoio produz também novas formas de compreensão já que muitas vezes o tratamento apresenta muitas dúvidas e angustias, fortalecendo não somente a livre expressão como também a tomada de consciência dos fatos apresentados. ${ }^{34}$

$\mathrm{O}$ uso da internet vem permeando como mecanismo de obtenção de informações sobre a doença e o tratamento, auxílio e apoio de familiares e amigos nas buscas na internet. É por meio dessa nova ferramenta tecnológica que os pacientes buscam novas formas de mudanças de pensamentos e ideias provocadas pelo diagnóstico. A rede oferta milhares de conhecimentos a respeito e podem conectar pessoas com as mesmas condições, compartilhando assim dicas, segredos, aflições e afins. É por meio dessas histórias de vida que é possível construir fontes de apoio para encarar as dificuldades não somente que o tratamento oferece, mas da vida de modo geral, como e por onde começar a reconstruir suas histórias assim como a construção e realização de novos sonhos e projetos de vida. $^{24}$

Estudos também apresentam que apesar da especificidade de cada caso, um dos fatores que previnem o aparecimento de qualquer tipo de câncer seria buscar um estilo de vida saudável, com prática de esportes, alimentação rica e balanceada. Outro ponto seria excluir alguns aspectos do cotidiano que agravam a saúde e contribuem para o surgimento do câncer, como a ingestão de bebidas alcoólicas e comidas não saudáveis, que facilita o aumento da massa corporal. ${ }^{36}$

Por meio das vivências de cada um é possível lidar com estímulos adversos que muitas provocam o adoecimento psíquico dos indivíduos. É nesse contexto que regiões físicas, como das partes cerebrais, 
também são afetas provocando um declínio no quadro de saúde, favorecendo o surgimento de patologias como o câncer. Desse modo é importante estar atento a todas as condições psicossociais a qual cada um encontra-se inserido, já que cada uma delas também é capaz de proporcionar não só um sofrimento psíquico como também corporal. ${ }^{37}$

Na perspectiva de adoecimento o sujeito passa a enfrentar uma série de desafios vindos do diagnóstico de câncer. Em muitos casos a hospitalização, de tempo variado, é necessária. Para isso as equipes de saúde nessas unidades se preparam para realizar ações que tenham cunho de cuidados paliativos, ou seja, promover atitudes que minimizem as adversidades vindas do processo de tratamento. Durante essas práticas, os profissionais precisam estar atentos a todas as dimensões as quais fazem parte da constituição dos pacientes. Esses dados se tornam importantes para um melhor entendimento das atitudes dos pacientes diante das situações de intervenções clínicas. ${ }^{39}$

Os cuidados paliativos não possuem como objetivo a curar do paciente, sua finalidade é proporcionar conforto ao paciente oncológico e amenizar seu sofrimento. Em alguns casos esse tratamento consegue dar ao doente uma aparente impressão de cura, com o aumento do alivio e a diminuição dos sintomas, que pode estar causando sofrimento físico, familiar, psicológico e espiritual para o paciente acometido pelo câncer. No contexto do tratamento paliativo em pacientes com câncer, podese perceber que a espiritualidade adquire diversos significados tanto para o paciente quanto para a equipe de saúde e os familiares do doente. O contexto espiritual também pode trazer mais tranquilidade para a equipe de enfermagem e a família do paciente oncológico. ${ }^{26}$

Para o paciente oncológico é importante que ele desenvolva resiliência, que se trata da capacidade de se recuperar de obstáculos que podem aparecer durante a vida, utilizando-se de força e resistência para superar as etapas do tratamento contra o câncer. A resiliência é uma maneira que a pessoa encontra para lidar com alguma situação problema, como um ponto favorável no tratamento do câncer. ${ }^{27}$ Os pacientes tendem a ter dificuldades de encarar os processos ligados ao adoecimento, esses processos podem influenciar na rotina da família do paciente e em suas próprias atividades diárias. A espiritualidade pode influenciar na recuperação do paciente ajudando, assim, o doente a encarar de forma melhor o processo do adoecimento e do tratamento. ${ }^{28}$

\section{A espiritualidade no enfrentamento do câncer}

Há várias questões ligadas ao uso da espiritualidade e da religiosidade no âmbito da medicina que devem ser levados em consideração durante o estudo do tema. Ao estudar a dor crônica e o uso de técnicas paliativas no tratamento da dor é muito importante levar em consideração aspectos da espiritualidade, religiosidade e fé. É necessário produzir mais pesquisas para definir com maior clareza o papel da espiritualidade/religiosidade no aspecto relacionado a dor sentida por eles durante o período de enfrentamento da doença. Desse modo é possível entender melhor o impacto da religiosidade na vida do paciente que utilizam essa alternativa junto ao tratamento clinico-hospitalar. ${ }^{29}$

A espiritualidade acaba por auxiliar no processo de ressignificação do câncer, causando uma motivação e aliviando possíveis medos ou crenças errôneas que possam surgir, já que o auxílio de uma força sobrenatural ou não se torna um importante aliado no combate ao sofrimento. Mas é importante ressaltar que a espiritualidade possa surgir após o impacto da doença, uma vez que estar mais próximo da presença da fé ameniza a sensação de estar mais próximo da morte. ${ }^{30}$ É pela via da espiritualidade que muitas vezes é possível encontrar possibilidades de intervenção que auxiliem no cuidado do paciente dentro do tratamento. A fé é capaz de reacender nas pessoas sentimentos como a esperança, algo fundamental dentro do quadro de hospitalização, uma vez que existem uma serie de medos sobre o câncer construídos e reforçados socialmente. ${ }^{38}$

Por meio da fé na espiritualidade adotada por cada um que advém forças que proporcionam o desejo de melhora através de pedidos nas orações. Os profissionais de saúde, como médicos, enfermeiros e psicólogos, compreendem esse aspecto como algo comum dentro do tratamento oncológico. É por meio dessas preces que se estabelecem um acalento para a alma diante de dias de insegurança e incerteza, trazendo dessa forma a fé como o único ponto seguro e "palpável" no imprevisível campo do adoecimento do por conta do câncer. ${ }^{33}$

Os pacientes entendem a religião/ espiritualidade como um suporte em momentos de dor, angústia e sofrimento, que se torna fundamental no doloroso processo de tratamento contra o câncer. 
Os pacientes oncológicos que utilizam dos benefícios da espiritualidade em seu tratamento costumam ver a comunidade religiosa como uma fonte de apoio, com a qual eles podem compartilhar suas aflições, seus sentimentos e até mesmo suas dores, assim eles se sentem confortados. Alguns doentes passam por uma transformação em seus valores, eles passam a ter valores que não tinha antes da doença e passam a agir e a viver de acordo com esses novos valores adquiridos. ${ }^{15}$

A espiritualidade oferta um cuidado não só espiritual como físico, uma vez que a fé possibilita a mudanças de pensamentos e crenças que trazem sofrimento ao sujeito, fornecendo subsídios para o cuidado da saúde física, proporcionando mais bem estar aos pacientes. Outro beneficio que a espiritualidade trás as pessoas é a redução de um adoecimento psíquico, diminuindo e em alguns casos ate extinguindo as possibilidades de doenças psicológicas que podem influenciar no tratamento oncológico, como por exemplo, a depressão, ansiedade e afins. ${ }^{35}$

Por meio das práticas que refletem a espiritualidade observam-se aspectos importantes da vida dos sujeitos, já que elas em sua grande parte são compartilhadas por indivíduos de locais distintos a qual o paciente faz isso, refletindo em uma prática comum cultural, reforçada por meio de usos e costumes. É nesse apoio que se constrói as bases importantes para o enfrentamento do câncer vindo do apoio espiritual como força, fé, esperança e afins. Portanto, é notório o aumento na frequência de estudos e pesquisas que reiteram esses dados, refletindo e contribuindo para a assimilação do fator espiritual dentro do processo saúde-doença, principalmente em patologias consideradas terminais. ${ }^{41}$

As práticas espirituais compartilhadas possibilitam a construção de uma rede de fé que se move em prol do paciente que se encontra em tratamento. Por meio dela é possível a construção de grupos que geram correntes de orações, que pedem não só o fortalecimento do sujeito, mas que possibilite a cura e o restabelecimento da saúde. E permita que a pessoa consiga a proteção necessária para lidar com os malefícios que ocorrem diante da luta contra o câncer. $^{44}$

Outro aspecto importante é a compreensão que a espiritualidade oferta sobre a morte e o morrer. Em muitos casos os pacientes se encontram fora de possibilidades terapêuticas, então esse fator produz a construção de novas representações sobre a morte, confortando muitas vezes e trazendo um alivio diante dos mistérios estabelecidos no pós morte. Busca-se por meio dela também novas compreensões sobre a vida, uma vez que se permite outros olhares sobre suas vivências e das relações que foram estabelecidas com outras pessoas. Há também um novo sentido dentro do morrer, propiciando um final de vida digno com menos procedimentos e situações que causam dor, desconforto e sofrimento. ${ }^{40}$

Como já destacado a espiritualidade fornece um apoio imensurável aos pacientes oncológicos, assim como para seus familiares e amigos queridos que muitas vezes participam de momentos do tratamento como cuidadores. Essa força ocasiona em momentos de reflexão onde as pessoas passam a questionar sobre qual o atual entendimento sobre sua vida, passando assim a sofrer modificações. Diante das atuações profissionais necessita-se de constantes estratégias de atualizações, para que esses trabalhadores ofertem melhores práticas diante do tratamento. ${ }^{43}$

Os profissionais da área da saúde devem estar preparados para abordar o paciente quanto a sua espiritualidade, sem ofensas e julgamentos, respeitando as preferências religiosas de cada doente, exercendo os cuidados em saúde de forma total e humanizada. Não existe apenas uma forma de abordar o paciente quanto a sua espiritualidade, muitas vezes essa abordagem é feita da forma mais natural possível. Quando a equipe de saúde tem bastante dificuldade de lidar com as convicções religiosas de seus pacientes, ela pode ter o auxílio de um líder religioso. Caso a equipe não consiga estabelecer um convívio harmonioso com o líder religioso, ela pode também recorrer a assistência psicológica ao disponível no hospital. $^{31}$

Um dos significados do cuidado espiritual é humanizar, ouvir de forma atenciosa, acalmar e está presente nos momentos de dor e sofrimento, mas para alguns pacientes a espiritualidade não interfere no processo de adoecimento e tratamento do câncer. As diferentes reações dos pacientes em relação ao enfrentamento do câncer estão ligadas as suas experiências intimas, religiosas, culturais e de acordo com os valores do próprio paciente. ${ }^{32}$ A clareza da importância desse ponto ao paciente oncológico permite que a equipe multidisciplinar, assim como a família, propicie uma melhor qualidade de vida, minimizando os impactos causados pelo câncer e estabelecendo praticas humanitárias que são 
embasadas por meio do respeito e a autonomia. ${ }^{40}$

Por meio da adoção dessas práticas que os profissionais conseguem estabelecer vinculo com os pacientes colaborando para a construção das etapas a serem seguidas no tratamento. É deste modo que se observa de fato o respeito ao sujeito por meio da livre demonstração da sua individualidade, considerando todas as crenças e visões pertinentes a cada um dentro do tratamento. ${ }^{42}$

\section{CONSIDERAÇÕES FINAIS}

O diagnóstico de câncer oferece um grande número de significados aos sujeitos. Essa notícia impacta não só o indivíduo assim como em todas as pessoas que possuem algum tipo de afetividade ligada a ela. $O$ câncer na maioria das vezes causa um impacto grande ao sujeito de tal forma que comumente este procura ressignificar toda sua maneira de vida, precisando se fortalecer usando como uma forma de enfretamento. Uma das estratégias mais utilizadas que se observou nessa pesquisa foi a maneira como eles buscam conhecer a doença. É neste momento de crise desencadeada pelo diagnóstico que a doença produz uma ressiginificação por meio da busca da espiritualidade como fator protetor.

A religião e a religiosidade são dois fatores diferentes, mas estão intimamente ligados e muitas vezes os significados dessas palavras se confundem. $\mathrm{O}$ indivíduo vai viver a sua religiosidade de acordo com o modo como encara a vida, pois cada pessoa possui uma experiência de vida diferente e isso influencia diretamente no modo como o se vive a religiosidade.

O tratamento contra o câncer costuma ser bastante agressivo e não só abala o paciente fisicamente como também o abala psicologicamente. A espiritualidade vem como uma alternativa paliativa na ajunta da dura batalha conta o câncer. A equipe de assistência em saúde que geralmente é formada por enfermeiros, médicos e psicólogos devem ter o conhecimento dessa alternativa de cuidado ao paciente. Nas últimas décadas a própria ciência vem conseguindo provar os efeitos benéficos do uso da religiosidade, muitos pacientes vêm comprovando melhoras em seu quadro clinico ao aderir a religiosidade junto ao tratamento convencional. Apesar das já comprovadas vantagens do uso da religiosidade, a ciência deve aprofundar mais ainda os seus estudos a respeito do tema.
A partir dessa discussão, podem-se compreender os significados e a importância das várias formas de enfrentamento ao tratamento do câncer, em especial a espiritualidade. Várias pesquisas apontam a espiritualidade como a forma de enfrentamento mais utilizada pelos pacientes oncológico. Em geral, é através da fé no divino, no sobrenatural, que os pacientes e, também seus cuidadores, se apoiam para todo o processo de dúvida, dor, medo, aceitação e encorajamento em todo o processo do tratamento.

\section{REFERÊNCIAS}

1. Brasil. Instituto Nacional de Câncer. ABC do câncer: Abordagens básicas para o controle do câncer. - Rio de Janeiro: Inca, 2011.

2. Brasil. Instituto Nacional de Câncer. O que é câncer. Rio de Janeiro. 2009. Disponível em: <http://http://www1.inca.gov.br/ conteudo_view.asp?id=322>. Acesso em: $21 \mathrm{dez} .2018$.

3. Organização Mundial de Saúde. Cáncer - Nota descriptiva. Disponível em: <http://www.who.int/mediacentre/factsheets/fs297/ es/>. Acesso em: 21 dez. 2018.

4. Brasil. Instituto Nacional de Câncer José Alencar Gomes da Silva. Estimativa 2016. - Rio de Janeiro: INCA, 2015. Disponível em: $<$ https://www.inca.gov.br/campanhas/dia-nacional-de-combateao-cancer/2015/estimativa-2016-incidencia-de-cancer-no-brasil>. Acesso em: 21 dez. 2018.

5. Geronasso MCH, Coelho D. A influência da religiosidade/ espiritualidade na qualidade de vida das pessoas com câncer. Saúde Meio Ambient 2012; 1(1):173-87. doi: https://doi.org/10.24302/ sma.v1i1.227

6. Costa P, Leite RCBO. Estratégias de Enfrentamento utilizadas pelos pacientes oncológicos submetidos a cirurgias mutiladoras. Revista Brasileira de Cancerologia 2009; 55(4): 355-64.

7. Bergamasco RB, Angelo M. Câncer de mama: Como o diagnóstico é experienciado pela mulher. Revista brasileira de cancerologia 2001;47(3):277-82

8. Sampaio AD, Siqueira HCH. Influência da Espiritualidade no Tratamento do Usuário Oncológico: Olhar da Enfermagem. Ensaios Cienc Cienc Biol Agrar Saúde 2016;20(3):153-60. doi: http:// dx.doi.org/10.17921/1415-6938.2016v20n3p153-160

9. Brasil. Instituto Brasileiro de Geografia e Estatística. Pesquisa nacional por amostra de domicílios, junho 2010. Rio de Janeiro.

10. Silva RR, Siqueira D. Espiritualidade, Religião e Trabalho no Contexto Organizacional. Psicologia em Estudo 2009;14(3):55764.

11. Machado FR, Zangari W, Maraldi EO, Martins LB, Shimabucuro AH. Contribuições da psicologia para a compreensão das relações entre a espiritualidade, a religiosidade e as experiências anômalas. Clareira-Revista de Filosofia da Região Amazônica 2016;3(2):2-21. 12. Kovács MJ. Espiritualidade e psicologia - cuidados compartilhados. Mundo da Saúde 2007;31(2):246-55.

13. Borges MDS, Santos MBC, Pinheiro TG. Representações sociais sobre religião e espiritualidade. Revista Brasileira de Enfermagem 2015;68(4):609-16.

14. Panzini RG, Rocha NSD, Bandeira DR, Fleck MPDA. Qualidade de vida e espiritualidade. Revista de psiquiatria clínica 2007;34(1)1:105-15.

15. Espíndola JA. O significado da religiosidade para pacientes com câncer e para profissionais de saúde. 2009. 234 p. Tese (Doutorado 
em Ciências) - Programa de Pós Graduação em Enfermagem Psiquiatrica da Escola de Enfermagem de Ribeirão Preto, Universidade de São Paulo, Ribeirão Preto, 2009.

16. Espíndola JA, Valle ERM, Bello AA. Religião e espiritualidade: um olhar de profissionais de saúde. Rev Latino-Am Enfermagem 2010;18(6):1229-36. doi: https://doi.org/10.1590/S010411692010000600025

17. Elmescany ENM, Barros MLP. Espiritualidade e Terapia Ocupacional: Reflexões em cuidados paliativos. Rev NUFEN 2015;7(2)1-24.

18. Schleder LP, Parejo LS, Puggina AC, Silva MJP. Espiritualidade dos familiares de pacientes internados em unidade de terapia intensiva. Acta Paulista de Enfermagem 2013; 26(1):71-8.

19. Angelo M. Ouvindo a voz da família: narrativas sobre sofrimento e espiritualidade. Mundo Saúde 2010;34(4):437-43.

20. Rother ET. Revisão sistemática $X$ revisão narrativa. Acta Paul Enferm 2007; 20(2). doi: http://dx.doi.org/10.1590/S010321002007000200001

21. Mattos K, Blomer TH, Campos ACBF, Silvério MR. Estratégias de enfrentamento do câncer adotadas por familiares de indivíduos em tratamento oncológico. Rev Psicol Saúde, 2016; 8(1):1-6. doi: http://dx.doi.org/10.20435/2177093X2016101

22. Nunes, CAC. O Conceito de Enfrentamento e Sua Relevância na Prática de Psicologia. Encontro: Revista Psicologia 2010;13(19):91102.

23. Rodrigues FSS, Polidori MM. Enfrentamento e Resiliência de Pacientes em Tratamento Quimioterápico e seus Familiares. Revista Brasileira de Cancerologia 2012;58(4):619-27.

24. Hey AP, Caveião C, Motezeli JH, Visentin A, Takano TM, Buratti, FMS. Meios de comunicação utilizados pelos pacientes: informações sobre o câncer após o diagnóstico e durante o tratamento Media used by patients: information about cancer after diagnosis and during treatment. Revista Online de Pesquisa: Cuidado é Fundamental 2016;8(3):4697-703. doi: http://dx.doi. org/10.9789/2175-5361.2016.v8i3.4697-4703

25. Costa P, Leite RCBO. Estratégias de enfrentamento utilizadas pelos pacientes oncológicos submetidos a cirurgias mutiladoras. Revista Brasileira de Cancerologia 2009;55(4):355-64.

26. Silva DIS. Significado e práticas da espiritualidade no contexto dos cuidados paliativos em pacientes oncológicos adultos. Revista HCPA 2011;31(3):353-8.

27. Rosa BVC, Girardon-Perlini NO, Begnin D, Rosa N, Stamm B, Coppetti LC. Resiliência em famílias de pessoas portadoras de colostomia por cancer: um olhar a partir do Sistema de crenças. Cienc Cuid Saude 2016;15(4):723-30. doi: http://dx.doi.org/10.4025/ cienccuidsaude.v15i4.34739

28. Soratto MT, Silva DM, Zugno PI, Daniel R. Espiritualidade e resiliência em pacientes oncológicos. Saúde e Pesquisa 2016; 9(1):5363. doi: http://dx.doi.org/10.177651/1983-1870.2016v9n1p53-63

29. Peres MFP, Arantes ACDLQ, Lessa PS, Caous CA. A importância da integração da espiritualidade e da religiosidade no manejo da dor e dos cuidados paliativos. Rev Psiq Clín 2007;34(1):82-7.

30. Cardoso AHA. Espiritualidade e câncer em pacientes submetidos a tratamento quimioterápico. 2014. 35 p. Monografia (Graduação em Enfermagem) - Universidade Estadual da Paraíba, Campina Grande. 2014.

31. Lucchetti G, Granero AL, Bassi RM, Latorraca R, Nacif SAP.
Espiritualidade na prática clínica: o que o clínico deve saber? Rev Bras Clin Med 2010; 8(2):154-8.

32. Pinto AC, Marchesini SM, Zugno PI, Zimmermann KG, Dagostin VS, Soratto MT. A importância da espiritualidade em pacientes com câncer. Rev Saúde Com 2015;11(2):114-22.

33. Bartolomei M. A fé como fator de resiliência no tratamento do câncer: uma análise do que pensam os profissionais da saúde sobre o papel da espiritualidade na recuperação dos pacientes. 2008. 137 p. Dissertação (Mestrado em Ciências da Religião). Pontifícia Universidade Católica de São Paulo - PUC-SP, São Paulo, 2008.

34. Hoffmann FS, Müller MC, Rubin R. A mulher com câncer de mama: apoio social e espiritualidade. Mudanças-Psicologia da Saúde 2006;14(2):143-50.

35. Miranda SL, Lara, MA, Felippe, WC. Espiritualidade, depressão e qualidade de vida no enfrentamento do câncer: Estudo exploratório. Psicologia: Ciência e profissão 2015;35(3): 870-85.

36. Inumaru LE, Silveira ÉAD, Naves MMV. Fatores de risco e de proteção para câncer de mama: uma revisão sistemática. Cadernos de Saúde Pública 2011;27(7):1259-70. doi: http://dx.doi. org/10.1590/S0102-311X2011000700002

37. Amorim MAP, Siqueira KZ. Relação entre vivência de fatores estressantes e surgimento de câncer de mama. Psicologia Argumento 2014;32(79):143-53. doi: http://dx.doi.org/10.7213/ psicol.argum.32.079.AO09

38. Sousa FFDPR, Freitas SMFM, Farias, AGS, Oliveira, MDCS, Araújo MFM, Veras VS. Enfrentamento religioso/espiritual em pessoas com câncer em quimioterapia: revisão integrativa da literatura. SMAD-Revista Eletrônica Saúde Mental Álcool e Drogas 2017; 13(1):45-51.

39. Arrieira, ICO, Thofern MB, Porto AR, Amestoy SC, Cardoso $\mathrm{DH}$. Espiritualidade e o processo de morrer: reflexões de uma equipe interdisciplinar de cuidados paliativos. Avances en Enfermería 2016;34(2):137-47.

40. Benites AC, Neme CMB, Santos MA. Significados da espiritualidade para pacientes com câncer em cuidados paliativos. Estudos de Psicologia 2017;34(2):269-79. doi: http://dx.doi. org/10.1590/1982-02752017000200008

41. Souza VM, Frizzo HCF, Paiva MHP, Bousso RS, Santos AS. Spirituality, religion and personal beliefs of adolescents with cancer. Rev Bras Enferm 2015;68(5):509-14. doi: http://dx.doi. org/10.1590/0034-7167.2015680504i

42. Souza ÉN, Oliveira NAD, Luchesi BM, Gratão ACM, Orlandi FDS, Pavarini SCI. Relação entre a esperança e a espiritualidade de idosos cuidadores. Texto \& Contexto-Enfermagem 2017;26(3):e6780015. doi: http://dx.doi.org/10.1590/010407072017006780015

43. Rocha RCNP, Pereira ER, Sora ABDA, Silva RMCRA. O impacto da espiritualidade frente às adversidades do câncer: revisão integrativa. Nursing 2016;19(218):1346-50.

44. Santos, IDL, Alvares RB, Lima NDM, Mattias SR, Cestari MEW, Pinto KRTDF. Câncer de mama: o apoio recebido no enfrentamento da doença. Rev Enferm UFPE 2017; 11(supl. 8), 3222-7.

Recebido em: 17/02/2019

Aceito em:10/06/2019

Como citar: SOUSA JUNIOR, Paulo de Tarso Xavier; TEIXEIRA, Selena Mesquita de Oliveira. A importância da espiritualidade no tratamento de pacientes oncológicos: uma revisão de literatura. Revista Interdisciplinar de Promoção da Saúde, Santa Cruz do Sul, v. 2, n. 1, jan. 2019. ISSN 2595-3664. Disponível em: <https://online.unisc.br/seer/index.php/ripsunisc/article/view/13195>. Acesso em: 02 jan. 2019. doi:https://doi.org/10.17058/rips.v2i1.13195 